\title{
Direct measurement of charged particle EDMs
}

\author{
C.J.G. Onderwater \\ on behalf of the Storage Ring EDM Collaboration
}

KVI, Zernikelaan 25, Groningen, the Netherlands

\begin{abstract}
Permanent electric dipole moments (EDMs) violate parity and time-reversal symmetry. Within the Standard Model (SM), they require CP violation and are many orders of magnitude below present experimental sensitivity. Many extensions of the SM predict much larger EDMs, which are therefore an excellent probe for the existence of 'new physics'. Until recently it was believed that only electrically neutral systems could be used for sensitive searches of EDMs. With the introduction of a novel experimental method, high precision for charged systems will be within reach as well. The features of this method and its possibilities are discussed.
\end{abstract}

\section{Introduction}

The study of the discrete symmetries $\mathcal{C}$ (charge conjugation), $\mathcal{P}$ (parity), and $\mathcal{T}$ (time reversal) has been the source of some of the most important progress in the understanding of fundamental interactions during the past 50 years. Whereas $\mathcal{C}$ and $\mathcal{P}$, and their product $\mathcal{C P}$, are conserved in strong ${ }^{1}$ and electromagnetic interactions, they are violated in the weak interaction. The (maximal) violation of $\mathcal{P}$ and $\mathcal{C}$ are well understood as a result of the handedness of the weak interaction, but the origin and magnitude of $\mathcal{C} \mathcal{P}$-violation (CPV) remains one of the most poorly understood features of the Standard Model.

The first evidence for CPV, in the neutral kaon system, was reported in 1964[1]. It took until 1973 before Kobayashi and Maskawa proposed a model that incorporates CPV through an irreducible complex phase in the weak-interaction quark mixing matrix[2]. This idea, which was presented at a time when only the $u, d$, and $s$ quarks were known to exist, was remarkable because it required the existence of six quarks, the last of which (the top-quark) was found in 1995. Only in $2001 \mathrm{CPV}$ was found outside the kaon system, viz.

\footnotetext{
${ }^{1}$ Strictly speaking, there is room for $\mathcal{C P}$ violation in the strong interaction through the $\bar{\theta}$ parameter. The latter is constrained to seemingly un-naturally small values.
} 
in the (decay of the) B-meson[3]. The observations were consistent with those predicted by the KM model, based on the earlier kaon measurements.

The baryon-antibaryon asymmetry in the universe (BAU) extracted from cosmological observations may provide another angle on CPV[4]. In his seminal paper, Sakharov showed that $\mathcal{C}$ and $\mathcal{C P}$ violation, together with non-conservation of baryon number and thermal non-equilibrium, may lead to a surplus of matter[6]. However, the amount of violation inferred from experiments using $\mathrm{K}$ and $\mathrm{B}$ mesons is insufficient to explain the cosmological asymmetry. For this and other reasons, the SM is believed to be incomplete, despite its enormous successes. Nowadays, leptonic $\mathcal{C} \mathcal{P}$-violation is also considered to be a possible route to explain the BAU. With the recent confirmation that neutrinos have non-zero mass ${ }^{2}$ (necessary for CPV), this route has gained plausibility.

Closely related to $\mathcal{C P}$-violation is the violation of time reversal symmetry $(\mathcal{T})$. To date, no undisputed experimental evidence exists of TRV (time-reversal violation). The CPLEAR collaboration claimed the first observation of direct TRV[7], but their results relies on the assumption that the combined $\mathcal{C P} \mathcal{T}$ symmetry is conserved. With this assumption, CPV is equivalent to TRV. As CPV has been established experimentally, this assumption requires that TRV should also occur at some level. However, when relaxing the requirement that $\mathcal{C P} \mathcal{T}$ is conserved, all claimed evidence is ambiguous regarding the violation of $\mathcal{C P} \mathcal{T}$ or $\mathcal{T}$. Similar observations were made by the $\mathrm{kTeV}$ collaboration[8].

Many searches are underway worldwide to set more stringent limits on TRV. Three possible observables include[9]

- triple correlations in $\beta$-decay, e.g. the D-coefficient that measures the strength of the term $\vec{J} \cdot\left(\vec{p}_{e} \times \vec{p}_{\nu}\right)$.

- permanent electric dipole moments on fundamental particles (and quantum systems with a non-degenerate ground state), i.e. $\vec{d} \cdot \vec{E} \neq 0$.

- "detailed balance" in scattering reactions, such as $p+n \rightleftharpoons d+\gamma$.

The search for detailed balance is conceptually most easily understood as a test of time reversal invariance, as indeed the final and initial states are exchanged. However, the search for detailed balance is severely hampered by experimental complexity. The scattering cross section is dominated by electromagnetic and possibly strong interactions, on top of which a small weak interaction perturbation could lead to TRV. It is therefore not considered as a viable route for precise studies of TRV.

Experiments trying to observe non-zero triple-correlation amplitudes include the measurement of the electron spin and momentum correlation in muon and neutron decay, and various nuclear $\beta$-decay experiments[10]. Without TRV, the amplitude of the triple correlation term must be zero. The same holds for permanent electric dipole moments (EDMs), which can only have a non-zero magnitude if both parity and time-reversal symmetry are broken. As with triple correlation measurements, there are multiple efforts underway worldwide to search for a non-zero EDM or to set more stringent limits. I will concentrate on EDMs in the remainder of this paper.

\footnotetext{
${ }^{2}$ The actual, indirect, evidence is the observation of neutrino oscillation, which requires non-zero neutrino masses. Neutrinoless double beta decay experiments also favor non-zero masses. Direct measurement of the anti-electron-neutrino mass is foreseen within the next decade at the KATRIN experiment[5].
} 


\section{The EDM landscape}

That permanent electric dipole moments break both $\mathcal{P}$ and $\mathcal{T}$ is manifest when considering the field dependent part of the interaction Hamiltonian $\mathcal{H}$ for a particle in an electric field $\vec{E}$ and a magnetic field $\vec{B}$,

$$
\mathcal{H}=-2(\vec{\mu} \cdot \vec{B}+\vec{d} \cdot \vec{E})=-2(\mu \vec{B}+d \vec{E}) \cdot \vec{S} .
$$

The second equality holds because the direction of the spin $\vec{S}$ is the only vector defining a direction in the rest frame of the particle. The electric and magnetic dipole moments must point along it; $\mu=g(e / m c)$ and $d=\eta / 2(e / m c)$ are the respective proportionality constants. Under parity, $\vec{E}$ reverses, while $\vec{B}$ and $\vec{S}$ remain unchanged. Similarly, for time reversal $\vec{B}$ and $\vec{S}$ reverse, while $\vec{E}$ remains unchanged. In both cases the Hamiltonian is altered, violating $\mathcal{T}$ and $\mathcal{P}$, unless $d=0$.

Within the Standard Model, the symmetry properties of $\mathcal{T}$ and $\mathcal{C P}$ are equivalent, which implies particles may acquire non-zero EDMs through $\mathcal{C P}$ violating radiative corrections of the electro-magnetic interaction vertex. To get non-canceling contributions, third or higher order loop corrections are necessary, which makes these EDMs extremely small and far below present detection limits[11]. In many proposed extensions of the SM, the need for higher-order is not present, and EDMs may occur even at first order[12]. With this in mind, the quest for a non-zero EDM is a very clear way to unambiguously establish the presence of physics beyond the Standard Model. In the case nothing is found, such searches will serve to rule out part of the space for new speculative models.

The topic of EDMs can be approached from several different and complementary angles. Each system is sensitive to different mechanisms that produce an EDM, as it gets its EDM in a hierarchical fashion[13]:

- quarks/leptons : may have intrinsic EDMs, which could mean inner structure, but they could also just have an EDM like they have a charge.

- nucleons : from quark EDMs or from quark gluon interactions.

- nuclei : from nucleon EDMs or nucleon nucleon (NN) force.

$-{ }^{2} \mathbf{H}$ : from nucleon EDMs or from the simplest two body NN force;

$-{ }^{3} \mathbf{H} /{ }^{3} \mathbf{H e}$ : many body NN interaction effects start to come in;

$-{ }^{6} \mathbf{L i}$ : real nuclear forces in action;

- heavy nuclei : strong enhancement of nucleonic and interaction effects; accessible with atomic physics methods (e.g. ${ }^{199} \mathrm{Hg}$ and ${ }^{2 x y} \mathrm{Ra}$ ).

- atoms : from nuclear EDMs, from electron EDMs, or from eN interactions; strong enhancement (paramagnetic atoms) or reduction (diamagnetic atoms) of sensitivity for constituent EDMs possible.

- molecules : very strong enhancement of constituent EDMs possible in polar molecules; increasing model dependence and uncertainty in extracting constituent EDMs.

Of this plethora of possibilities, only a few have been explored (see Table 1). 
Table 1: Overview of the most stringent experimental EDM limits (95\% C.L.), the system in which they were measured, the Standard Model value and the largest, allowed, prediction from various SM extensions.

\begin{tabular}{ccccc}
\hline particle & limit $[\mathrm{e} \cdot \mathrm{cm}]$ & system & SM $[\mathrm{e} \cdot \mathrm{cm}]$ & New Physics $[\mathrm{e} \cdot \mathrm{cm}]$ \\
\hline electron & $1.9 \times 10^{-27}$ & ${ }^{205} \mathrm{Tl}$ atom & $\sim 10^{-38}$ & $10^{-27}$ \\
muon & $9.3 \times 10^{-19}$ & rest frame E field & $\sim 10^{-35}$ & $10^{-22}$ \\
tau & $2.5 \times 10^{-17}$ & $\left(e^{+} e^{-} \rightarrow \tau^{+} \tau^{-} \gamma^{*}\right)$ & $\sim 10^{-34}$ & $10^{-20}$ \\
proton & $5.4 \times 10^{-24}$ & ${ }^{199} \mathrm{Hg}$ atom & $\sim 10^{-31}$ & $5 \times 10^{-26}$ \\
neutron & $7.4 \times 10^{-26}$ & ultra cold neutrons & $\sim 10^{-31}$ & $5 \times 10^{-26}$ \\
${ }^{199} \mathrm{Hg}$ & $2.1 \times 10^{-28}$ & ${ }^{199} \mathrm{Hg}$ atom & $\sim 10^{-33}$ & $10^{-28}$ \\
\hline
\end{tabular}

The experimental signature of an EDM is the dependence of the spin precession frequency on the strength of an externally applied electric field. For a precise measurement, both the electric field felt by the particle under study and the observation time need to be as large as possible. For atomic systems the first condition is usually not met for its constituents. For example, the rearrangement of the atomic electrons under the influence of the external electric field shields the nucleus from this field[14]. Only large and heavy nuclei, such as ${ }^{199} \mathrm{Hg}$, have a sufficiently large so-called Schiff moment to allow experimental access[15]. Because of relativistic effects, the shielding for the electrons is also not complete. Both the nuclear EDM and electron EDM can thus contribute to the atomic EDM. The more fundamental EDMs of the nucleus, the electrons and possibly their interaction must be inferred using theoretical models[16].

Direct precise measurement of the EDM of a charged particle is complicated because of the necessary strong electric fields. Charged particles accelerate out of the experiment, preventing a long observation time. Recently, a novel experimental method was proposed, that circumvents these problems and allows the EDM of charged particles to be measured directly[17]. This method provides direct access to the very interesting realm of light nuclei, which so far have not been examined for EDMs. Since its conception, several improvements and extensions have been made. Below I will outline the most prominent features of this technique and previous measurements of charged particle EDMs.

\section{Use of magnetic storage rings}

Magnetic storage rings and Penning traps have been used to precisely measure magnetic dipole moments, notably of the electron and muon[18, 19]. In the absence of an electric dipole moment, the spin evolution is described by the so-called BMT or Thomas equation,

$$
\frac{d \vec{S}}{d t}=\frac{e}{m c} \vec{S} \times\left[a\left(\vec{B}-\frac{\gamma}{\gamma+1}(\vec{\beta} \cdot \vec{B}) \vec{\beta}-\vec{\beta} \times \vec{E}\right)+\frac{1}{\gamma} \vec{B}-\frac{1}{\gamma+1} \vec{\beta} \times \vec{E}\right]
$$

with the magnetic moment anomaly $a=(g-2) / 2$, with $g$ as defined in section 2 . This equation simplifies considerably, if we consider the spin in a reference frame that rotates 
along with the particle as it goes around in a storage ring. Secondly, the magnetic field is usually orthogonal to the velocity, so that $\vec{\beta} \cdot \vec{B}$. In this case, eq. (2) simplifies to

$$
\frac{d \vec{S}}{d t}=\frac{e}{m c} \vec{S} \times\left[a \vec{B}+\left(a-\frac{1}{\gamma^{2}-1}\right) \vec{\beta} \times \vec{E}\right] .
$$

For extremely low velocities, such as in a Penning trap, or for the 'magic momentum' $\left(a-\frac{1}{\gamma^{2}-1}\right)=0$, this simplifies even further to $\frac{d \vec{S}}{d t}=\frac{a e}{m c} \vec{S} \times \vec{B}$.

When a non-zero EDM is assumed some additional terms appear,

$$
\frac{d \vec{S}}{d t}=\frac{e}{m c} \vec{S} \times\left[a \vec{B}+\left(a-\frac{1}{\gamma^{2}-1}\right) \vec{\beta} \times \vec{E}+\frac{\eta}{2}(\vec{E}+\vec{\beta} \times \vec{B})\right] \equiv \vec{S} \times \vec{\Omega} .
$$

Several techniques can be devised to gain sensitivity to the EDM, three of which are outlined below.

\subsection{The 'parasitic' way}

For relativistic particles, for which $\beta \gg 0$, the motional electric field $\vec{\beta} \times \vec{E}$ in the rest frame of the particle may be significant. Both the magnetic and electric dipole moments contribute to the precession $\vec{\Omega}=\vec{\omega}_{a}+\vec{\omega}_{\eta}$. With the assumption that the average electric field in the laboratory system is zero, $\vec{\omega}_{a}=(a e / m c) \vec{B}$ and $\vec{\omega}_{\eta}=(\eta e / 2 m c)(\vec{\beta} \times \vec{B})$. These two components are orthogonal. The magnitude of the precession rate therefore depends on the EDM in second order,

$$
\Omega=\sqrt{\omega_{a}^{2}+\omega_{\eta}^{2}} \simeq \omega_{a}\left[1+\frac{1}{2}\left(\frac{\eta \beta}{2 a}\right)^{2}\right]
$$

Furthermore, the precession plane, defined by the vector $\vec{\Omega}$, is tilted with respect to the plane containing the orbits of the particles, defined by $\vec{B}$. The tilt angle is

$$
\phi=\arctan \frac{\omega_{\eta}}{\omega_{a}} \simeq \frac{\eta \beta}{2 a} .
$$

Note that this tilt is always radially inward or outward.

At this point, two methods can be identified to search for an EDM. First, one could very precisely measure the spin precession rate and account for the contribution from the anomalous magnetic moment, which would have to be calculated, or obtained by other means. Precise calculations are only feasible for structureless particles, i.e. leptons, and even then the most precise calculation has a precision of a few parts-per-billion (for the electron). As the EDM contributes only to second order, this precision is by far not enough for a competitive experiment on the electron. It should be noted though, that in the presence of an EDM the rate of precession always goes up. Model dependence can be removed by measuring the spin precession rate as a function of $\beta$.

The tilt in the precession plane gives a more solid and unambiguous handle on the EDM. Assuming that the initial polarization is entirely along the momentum, the components 
pointing along the magnetic field (the EDM signal), pointing sideways and along the momentum (the anomalous magnetic moment signal), are respectively given as

$$
\begin{array}{rlrl}
P_{\|}(t) & =P_{\circ} \frac{\omega_{\eta}}{\sqrt{\omega_{a}^{2}+\omega_{\eta}^{2}}} \sin \sqrt{\omega_{a}^{2}+\omega_{\eta}^{2}} t & \simeq P_{\circ} \frac{\eta \beta}{2 a} \sin \Omega t \\
P_{\perp}(t)=P_{\circ} \frac{\omega_{a}}{\sqrt{\omega_{a}^{2}+\omega_{\eta}^{2}}} \sin \sqrt{\omega_{a}^{2}+\omega_{\eta}^{2}} t & \simeq P_{\circ} \sin \Omega t \\
P_{\rightleftharpoons}(t)=P_{\circ} \cos \sqrt{\omega_{a}^{2}+\omega_{\eta}^{2}} t & \simeq P_{\circ} \cos \Omega t
\end{array}
$$

The last equalities hold to first order in $\eta$. In the absence of an EDM, the spin component along the magnetic field cannot be time dependent. The challenge lies in the determination of the orientation of the (effective) magnetic field. The only reported measurements of this kind have been made on the muon at CERN and later at Brookhaven[20,21], with $d_{\mu}<2.8 \times 10^{-19} \mathrm{e} \cdot \mathrm{cm}(95 \%$ C.L. $)$ as the final limit for the muon EDM.

The sensitivity of this method is limited, because the vertical polarization component can only grow for a time of the order of the precession period. The growth-time can be significantly increased if the contribution of the magnetic moment to the spin precession is reduced.

\subsection{Frozen spin method}

The sensitivity for an EDM is increased if $\omega_{a} \rightarrow 0$ (see eq. (6)), in which case the growth of the vertical polarization component continues as long as the beam is polarized. The use of a radially oriented electric field was proposed in Ref. [17]. In the presence of an electric field, the contributions of the magnetic and electric dipole moments become

$$
\vec{\omega}_{a}=\frac{e}{m c}\left[a \vec{B}+\left(a-\frac{1}{\gamma^{2}-1}\right) \vec{\beta} \times \vec{E}\right] \quad \vec{\omega}_{\eta}=\frac{e}{m c} \frac{\eta}{2}(\vec{\beta} \times \vec{B}+\vec{E})
$$

For a field strength given by

$$
E_{r}=\frac{a B c \beta}{1-(1+a) \beta^{2}} .
$$

$\omega_{a}$ can be made zero. The time evolution of the polarization becomes

$$
\begin{aligned}
P_{\|}(t) & =P_{\circ} \sin \omega_{\eta} t \simeq P_{\circ} \frac{\eta e}{4 m c}\left(\beta B+E_{r}\right) t \\
P_{\perp}(t) & =0 \\
P_{\rightleftharpoons}(t) & =P_{\circ} \cos \omega_{\eta} t \simeq P_{\circ}
\end{aligned}
$$

The EDM thus manifests itself as a linearly growing vertical polarization component.

The strengths of this method lie in the fact that the growth time of the vertical spin component can be as long as the spin coherence time of the beam and that the electric field in the particle rest frame may be significantly larger than that applied in the laboratory frame, by a factor

$$
\xi=\frac{\beta B_{z}+E_{r}}{E_{r}}=\frac{a+1}{a \gamma^{2}}
$$


For particles with small $a$ and low $\gamma$ this amplification can be of the order of 10-100.

The statistical precision of this method depends on several factors, such as the initial polarization of the beam $P_{\circ}$, the beam lifetime $\tau$, the polarization coherence time $\tau_{p}$, the strength of the electric field in the center of mass $\beta c B_{z}-E_{r}$, the analyzing power of the polarimeter $A$, and the number of particles used to make the measurement $N_{\text {tot }}$. The uncertainty then can be described in good approximation by

$$
\sigma_{d} \simeq \frac{4 \hbar}{\sqrt{\tau \tau_{p}}\left(\beta c B_{z}-E_{r}\right) P_{\circ} A \sqrt{N_{t o t}}}
$$

Table 2: Characteristic storage ring features for EDM experiments on the muon and deuteron. Statistical precision is for $N=10^{16}$ particles.

\begin{tabular}{lccccccccc}
\hline & $a$ & $\begin{array}{c}p \\
{[\mathrm{GeV} / c]}\end{array}$ & $\gamma$ & $\xi$ & $\begin{array}{c}E \\
{[\mathrm{MV} / \mathrm{m}]}\end{array}$ & $\begin{array}{c}B \\
{[\mathrm{~T}]}\end{array}$ & $\begin{array}{c}R \\
{[\mathrm{~m}]}\end{array}$ & $\begin{array}{c}\tau \\
{[\mathrm{s}]}\end{array}$ & $\begin{array}{c}\sigma_{d} \\
{[\mathrm{e} \cdot \mathrm{cm}]}\end{array}$ \\
\hline$\mu$ & 0.001 & 0.5 & 5 & 34 & 2 & 0.25 & 7 & $10^{-5}$ & $10^{-24}$ \\
${ }^{2} \mathrm{H}$ & -0.143 & 0.7 & 1.06 & -5.5 & 3.5 & 0.21 & 13 & 10 & $10^{-27}$ \\
\hline
\end{tabular}

The storage ring must be designed with special care to reach a long spin coherence time. This includes the use of higher order magnetic elements, such as sextupoles. The momentum spread is one of the most significant sources for depolarization. Limiting the momentum acceptance reduces the intensity of the beam and is therefore not preferred. A possible solution is to use an RF-cavity to set up synchrotron oscillations, i.e. an oscillation of the momentum. The frequency of the cavity defines the revolution frequency of the particles in the ring and thus the average momentum. The deviation of the initial momentum translates into a synchrotron phase. Now the spread in the average momentum contributes to the depolarization of the beam, rather than the initial momentum spread.

The dominant source of systematic uncertainty for this technique is an electric field component parallel to the magnetic field. In the rest frame spin precession about the resulting radial magnetic field component $\vec{B}_{r}^{*}=\vec{\beta} \times \vec{E}_{z}$ mimics an EDM signal. When injecting the beam clockwise and counter-clockwise the sign of the signal is opposite, since $\beta$ changes sign, but $E$ (and thus $E_{z}$ ) remains the same.

One of the challenges of this method is the limited strength of the radial electric field. Therefore, this technique is only suited for particles with small anomalous magnetic moments. Also, because of the limited electric field strength and linked magnetic field strength, see eq. (9), the setup may become rather large.

\subsection{Spin resonance}

The limitations imposed by the radial electric field can be avoided by yet another technique. From eq. (6), it follows that the tilt angle does depend on the velocity. The spin 
motion is described by

$$
\begin{aligned}
\frac{d \vec{S}}{d t} & =\vec{S} \times \frac{e}{m c}\left[a B+\eta\left(\overrightarrow{\beta_{0}} \times \vec{B}\right)+\eta \cos (\omega t+\phi)\left(\delta \vec{\beta} \times \vec{B}+\vec{E}_{R F}\right)\right] \\
& \equiv \vec{S} \times[\vec{\Omega}+\eta \delta \vec{\Omega} \cos (\omega t+\phi)]
\end{aligned}
$$

Because $\Omega \gg d \Omega$, the oscillating term can be treated as a perturbation. The vertical polarization component is given by

$$
\left.d P_{\|} / d t=\eta P_{\circ} \delta \Omega \cos (\Omega t+\phi) \cos (\omega t+\psi) \simeq \frac{1}{2} \eta P_{\circ} \delta \Omega \cos (\Delta \omega t+\Delta \phi)\right) .
$$

In the last step, fast oscillating terms $(\propto \cos ((\Omega+\omega) t))$ were omitted and it was assumed that $\Delta \omega \equiv \Omega-\omega \simeq 0$ and $\Delta \phi \equiv \phi-\psi \simeq 0$. For $\Delta \omega=0$ the vertical polarization will grow continuously at a rate proportional to the EDM. Maximum sensitivity is obtained for $\Delta \phi=0$ and $\Delta \phi=\pi$. In the latter case the sign of the growth is reversed.

The RF cavity introduced in the previous section sets up free and thus incoherent synchrotron oscillations. For the method described here, the oscillation must be coherent. This can be achieved by using the first cavity to bring the beam close to resonance. A second cavity is then used to drive the beam oscillations.

The statistical sensitivity of this method is similar to that for the 'frozen spin' method. In the latter, the EDM signal was proportional to $\vec{v} \times \vec{B}$. The value of $B$ is limited because of the limitations imposed by the freezing condition and maximum electric field strength. In this method, no such limit is present, and the magnetic field strength can be raised as high as practical. So although the uncertainty is proportional to the amplitude of the velocity modulation $d v$, rather than $v$, the product $d \vec{v} \times \vec{B}$ is similar is size.

In the 'frozen spin' method static perturbations of the experiment could produce signals mimicking an EDM, since the overall spin precession rate was close to zero. In the resonance method, the spin precesses at a very high rate. This decouples any (near) static perturbations from the spin.

This method is suitable for all particles for which the synchrotron tune $\Omega_{L}$ and spin tune $\Omega_{\text {spin }}$ can be brought to resonance, i.e. $\Omega_{L}-\Omega_{\text {spin }}=n$ ( $n$ integer). The spin tune is given by $\Omega_{\text {spin }}=a \gamma$. The synchrotron tune is proportional to the square root of the gradient in the cavity, which is in turn determined by the harmonic number and operating voltage of the cavity. The latter will be the limiting factor for particles with relatively large anomalous magnetic moments. The method was initially aimed at the search for a deuteron EDM $(a=-0.143)$. Presently, it is believed that also other light ion EDMs might be within reach, such as the proton $(a=1.79)$ and ${ }^{3} \mathrm{He}(a=-4.19)$. Although theoretical work is still in progress[22], the latter is expected to be mostly sensitive to the neutron EDM. Together with a measurement of the proton EDM and the deuteron EDM (which is mostly due to the nuclear interaction), a very significant contribution to the field can be made. 


\section{Conclusion and Outlook}

Three methods were described to directly measure permanent electric dipole moments of charged particles. All methods make use of the strong motional electric field relativistic particles experience while moving in a magnetic storage ring. In the rest frame of the particle, the coupling of the magnetic and electric dipole moments to the magnetic and electric fields causes the spin to precess.

To date, the only direct measurement of a charged particle EDM that reaches reasonable precision $\left(d_{\mu}<\mathcal{O}\left(10^{-19}\right) \mathrm{e} \cdot \mathrm{cm}\right)$ was made for the muon, in an experiment aimed to measure the anomalous magnetic moment. The EDM was extracted from the tilt in the spin precession plane. The sensitivity of this method is limited by systematic uncertainties.

A first step towards increased sensitivity can be made by 'freezing' the precession caused by the anomalous magnetic moment, using a radially oriented electric field. It is expected that in a dedicated experiment, the EDM measurement on the muon can be improved by some five orders of magnitude. The method of frozen spin is applicable for all particles with a sufficiently small anomalous magnetic moment, the magnitude of which determines the strength of the radial electric field. The largest systematic uncertainty for this method is likely to originate from a vertical electric field component. For the deuteron (one of the candidate light ions), this systematic error is estimated to be of the order of $10^{-27} \mathrm{e} \cdot \mathrm{cm}$.

The second step is to remove this large systematic error. By setting up a spinsynchrotron resonance, the radial electric field is no longer needed. Because of the residual spin precession, static perturbations decouple from the EDM signal. EDM limits of the order of $10^{-29} \mathrm{e} \cdot \mathrm{cm}$ seem reachable with this method.

Already at a sensitivity of $10^{-24} \mathrm{e} \cdot \mathrm{cm}$, which seems readily achievable, several speculative new physics models can be tested[23]

\section{Acknowledgments}

We want to thank our colleagues from the ring-EDM collaboration for their valuable input to this writeup. The ideas presented here are mainly conceived in their brains. The research described in this paper was partly financed by an Innovative Research grant from the Dutch Organization for Fundamental Research.

\section{References}

[1] J.H. Christenson, J.W. Cronin, V.L. Fitch, and R. Turlay, Phys. Rev. Lett. 13, 138 (1964)

[2] M. Kobayashi and T. Maskawa, Prog. Theor. Phys. 49, 652 (1973).

[3] B. Aubert, et al. [Babar Collaboration], Phys. Rev. Lett. 87, 091801 (2001); K. Abe, et al. [Belle Collaboration], Phys. Rev. Lett. 87, 091802 (2001).

[4] V. Barger, J.P. Kneller, H-S. Lee, D. Marfatia and G. Steigman, Phys. Lett. B566 (2003) 8.

[5] A. Asipowics et al., hep-ex/0109033 (2001).

[6] A.D. Sakharov, Pis'ma Zh. Eksp. Teor. Fiz. 5, 32 (1967) [JETP Lett. 5, 24 (1967)]. 
[7] A. Angelopoulos, et al.[CPLEAR collaboration], Phys.Lett. B444 (1998) 43; for comments see e.g. A. Rouge, arXiv.org:hep-ph/9909205, I.I. Bigi and A.I.Sanda, arXiv.org:hep-ph/9904484

[8] A. Alavi-Harati et al.[kTEV collaboration], Phys. Rev. Lett. 84, 408 (2000).

[9] For a review, see e.g. P. Herczeg, Prog. Part. and Nucl. Phys. 46, 413 (2001).

[10] various experiments such as, e.g., muPT @ PSI (http://ares.ethz.ch/mupt/), emiT @ NIST (http://physics.nist.gov/Divisions/Div846/Gp3/FunPhys/emitexpt.html), TRI $\mu \mathrm{P} @$ KVI (http://www.kvi.nl/ trimp/web/html/trimp.html)

[11] see e.g. I.B. Khriplovich and S.K. Lamoreaux, CP Violation Without Strangeness, Springer Verlag (1997).

[12] see e.g. P.G.H. Sandars, Contemp. Phys. 42 (2001) 97.

[13] see e.g. C.R. Gould and E.D. Davis, "Time reversal invariance in nuclear physics: from neutrons to stochastic systems", in CP Violation in Particle, Nuclear and Astrophysics, Michael Beyer (Ed.), Springer Verlag (2002) 207.

[14] E.M. Purcell and N.F. Ramsey, Phys. Rev. 78 (1950) 807.

[15] L.I. Schiff, Phys. Rev. 132 (1963) 2194.

[16] see e.g. J.M. Pendlebury and E.A. Hinds, Nucl. Instr. Meth. A440 (2000) 471.

[17] F.J.M. Farley et al., Phys. Rev. Lett. 93 (2004) 052001.

[18] R.S. Van Dyck, Jr., P.B. Schwinberg, and H.G. Dehmelt Phys. Rev. Lett. 59, 2629 (1987)

[19] G.W. Bennett et al., Phys. Rev. Lett. 89 (2002) 101804; G.W. Bennett et al., Phys. Rev. Lett. 92 (2004) 161802; and references therein.

[20] J. Bailey et al. [CERN Muon Storage Ring Collaboration], J. Phys. G 4 (1978) 345.

[21] R. McNabb [Muon g-2 Collaboration], arXiv:hep-ex/0407008.

[22] R.G.E. Timmermans and C.-P. Liu, private communication.

[23] M. Pospelov and A. Ritz, arXiv:hep-ph/0504231, to appear in Annals of Physics. 